INPLASY

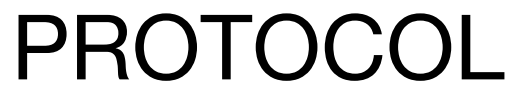

To cite: Azab et al. Has the COVID-19 pandemic affected the management of brain tumours? A systematic review and meta-analysis protocol. Inplasy protocol 202080082. doi:

10.37766/inplasy2020.8.0082

Received: 19 August 2020

Published: 19 August 2020

Corresponding author:

Ahmed Y Azzam

ahmedyazzam@gmail.com

Author Affiliation:

October 6 University Faculty of Medicine

Support: Not Available.

Review Stage at time of this submission: Preliminary searches.

Conflicts of interest:

No Conflict of Interest.

\section{Has the COVID-19 pandemic affected the management of brain tumours? A systematic review and meta-analysis protocol}

\author{
Azab, MA¹; Ghozy, S2; Alomari, A33; Azzam, AY4.
}

Review question / Objective: This review seeks to follow-up on the management lines have done with brain tumours cases during the period of December 2019 to August 2020 during the COVID-19 pandemic according to the infection status of the patient and status of the surgery.

Condition being studied: Management lines of brain tumours cases including (Glial tumours, Pituitary adenoma, CPA lesions, Meningioma, Haemangioblastoma, CNS lymphoma, Craniopharyngiomas, Pineal tumours, Brain Metastases) during the period of December 2019 to August 2020 during the COVID-19 pandemic.

INPLASY registration number: This protocol was registered with the International Platform of Registered Systematic Review and Meta-Analysis Protocols (INPLASY) on 19 August 2020 and was last updated on 19 August 2020 (registration number INPLASY202080082).

\section{INTRODUCTION}

Review question / Objective: This review seeks to follow-up on the management lines have done with brain tumours cases during the period of December 2019 to August 2020 during the COVID-19 pandemic according to the infection status of the patient and status of the surgery.

Condition being studied: Management lines of brain tumours cases including (Glial tumours, Pituitary adenoma, CPA lesions, Meningioma, Haemangioblastoma, CNS 
lymphoma, Craniopharyngiomas, Pineal tumours, Brain Metastases) during the period of December 2019 to August 2020 during the COVID-19 pandemic.

\section{METHODS}

Search strategy: (Glioblastoma AND COVID-19) OR (Glioblastoma AND SARSCOV-2) OR (Glioma AND COVID-19) OR (Glioma AND SARS-COV-2) OR (Glial AND COVID-19) OR (Glial AND SARS-COV-2) OR (Astrocytoma AND COVID-19) OR (Astrocytoma AND SARS-COV-2) OR (CPA lesions AND COVID-19) OR (CPA lesions AND SARS-COV-2) OR (Pituitary adenoma AND COVID-19) OR (Pituitary adenoma AND SARS-COV-2) OR (Acoustic neuroma AND COVID-19) OR (Acoustic neuroma AND SARS-COV-2) OR (Meningioma AND COVID-19) OR (Meningioma AND SARSCOV-2) OR (Oligodendrogliomas AND COVID-19) OR (Oligodendrogliomas AND SARS-COV-2) OR (Haemangioblastoma AND COVID-19) OR (Haemangioblastoma AND SARS-COV-2) OR (CNS lymphoma AND COVID-19) OR (CNS lymphoma AND SARS-COV-2) OR (Ependymomas AND COVID-19) OR (Ependymomas AND SARSCOV-2) OR (Schwannomas AND COVID-19) OR (Schwannomas AND SARS-COV-2) OR (Craniopharyngiomas AND COVID-19) OR (Craniopharyngiomas AND SARS-COV-2) OR (Pineal tumours AND COVID-19) OR (Pineal tumours AND SARS-COV-2) OR (Brain Tumours AND COVID-19) OR (Brain Tumours AND SARS-COV-2) OR (Brain Metastases AND COVID-19) OR (Brain Metastases AND SARS-COV-2).

Participant or population: All eligible brain tumours cases.

Intervention: All neurosurgical operations for brain tumours cases.

\section{Comparator: Not Applicable.}

Study designs to be included: All (no exclusion based on study design).

Eligibility criteria: Articles for brain tumours cases between December 2019 to August 2020 during the COVID-19 Pandemic.
Information sources: Multiple databases will be searched, including, PubMed, MEDLINE, Scopus, and Google Scholar.

Main outcome(s): To follow-up on the management lines that have been done with brain tumours cases during the period of December 2019 to August 2020 amid the COVID-19 pandemic according to the infection status of patients and status of surgery.

Additional outcome(s): Not planned yet.

Quality assessment / Risk of bias analysis: Cochrane's revised quality assessment tool (RoB 2) was used to determine the quality of the randomized trials, whereas ROBINS-I tool was used to assess the quality of nonrandomized ones.

Strategy of data synthesis: All statistical analyses will be done using $R$ software version 4.0.2 and "meta" statistical package. Dichotomous data will be presented as odds ratio (OR) with 95\% confidence interval $(\mathrm{Cl})$ and continuous data will be reported as mean difference (MD) with $95 \% \mathrm{Cl}$. Data analysis will be done using a fixed-effects or randomeffects, according to heterogeneity level. Heterogeneity will be assessed with $Q$ statistics and 12 test considering it significant with 12 value $>50 \%$ or $\mathrm{P}$-value < 0.05 . If present, a baujat plot will be used to detect the main source of heterogeneity. We will perform Begg's funnel plot and Egger's regression test to evaluate the presence of publication bias when there were ten or more studies in the analysis. The statistical significance will be considered at $p$-value $<0.1$ (two-tailed test). If the publication bias was found, the trim and fill method of Duvall and Tweedie will be performed by adding studies that appeared to be missing to enhance the symmetry.

Subgroup analysis: Patients will be stratified according to the infection status of the patient and status of the surgery.

Sensibility analysis: No sensitivity analysis will be conducted. 
Language: English articles only.

Country(ies) involved: Egypt.

Keywords: Brain tumours; CNS; CNS Tumours; COVID-19; SARS-CoV-2.

Dissemination plans: The results of this study will be summarized in English and disseminated by submission for publication in a peer-reviewed journal.

Contributions of each author:

Author 1 - Mohammed A Azab Conceptualization, Investigation, Methodology, Writing - Original Draft.

Author 2 - Sherief Ghozy - Formal analysis, Data Curation, Visualization, Writing Review \& Editing.

Author 3 - Abdulraheem Alomari Validation, Resources, Writing - Review \& Editing.

Author 4 - Ahmed Y Azzam - Supervision, Investigation, Validation, Writing - Review \& Editing. 